\title{
La qualité doit être au service de l'efficacité
}

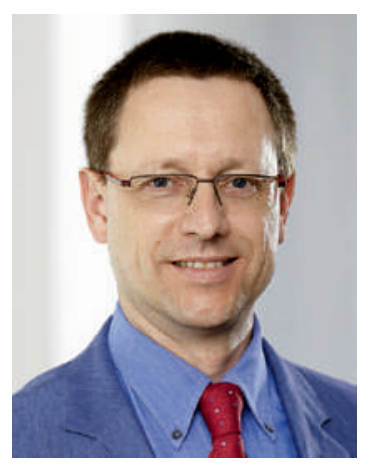

Comme à chaque fois que je suis confronté à une question difficile, j'ai demandé conseil à mes enfants, qui m'ont répondu sans hésiter: «La qualité, c'est quand j'obtiens ce que je veux.» La qualité n'est donc pas un terme absolu mais une notion relative, dont la définition varie selon les intérêts, les attentes et le rôle de chacun. En matière de qualité, l'ampleur et la priorité qu'on lui confère différent également.

Mais c'est précisément cette pluralité des approches qui est enrichissante et qui nous permet, en fin de compte, d'éviter de nous cantonner dans une position trop unidimensionnelle. La question de la qualité nous confronte à nos limites en matière de définitions et de procédures: ce qui est bon pour

\section{La qualité n'est pas une notion absolue. Elle diffère en fonction des intentions et des objectifs.}

l'un ne l'est pas nécessairement pour tous; ce qui est bien dans une situation donnée ne l'est ni toujours ni partout. Après tout, je n'utilise pas le même thermomètre pour mesurer la température de mon rôti et pour mesurer celle de la neige afin de choisir la couleur de mon fart...

Dans le domaine de la santé, l'administration (fédérale ou cantonale) et les milieux politiques ont souvent recours au concept de qualité. Dans la LAMal par exemple, les fournisseurs de prestations sont tenus de communiquer les indicateurs de qualité médicaux. Mais la vision de l'Etat qui, selon la Constitution, doit veiller à une couverture médicale suffisante n'est pas la même que celle des assureurs, qui doivent fournir les moyens financiers. Et la vision des médecins, qui s'attachent à atteindre, avec leurs patients, un objectif formulé de manière individuelle en fonction du problème de santé du moment, est encore différente.

\section{Le Swiss Quality Award se concentre sur des projets qui profitent aux patients et font avancer la gestion de la qualité dans le domaine de la santé.}

Néanmoins, la question de la qualité fait des émules. A l'instar des lauréats du Swiss Quality Award 2014, remis par l'Institut pour la recherche évaluative en médecine (IEFM) de l'Université de Berne, la Société suisse pour la gestion de la qualité dans le domaine de la santé (SQMH) et la FMH. Pour la première fois cette année, le prix a été remis dans les nouvelles catégories Secteur ambulatoire, Secteur hospitalier et Projet intersectoriel, et le meilleur poster a été récompensé par le jury dans le cadre du Poster-Award.

Les projets récompensés montrent parfaitement qu'avec des objectifs clairement formulés, des fondements et des processus transparents, les démarches en faveur de la qualité sont un gage de succès - et ce toujours dans l'intérêt des patientes et des patients. Je profite de ces quelques lignes pour féliciter les trois équipes lauréates et pour remercier, au nom des organisations partenaires, tous les participants au Swiss Quality Award de cette année pour leurs remarquables projets.

Dr Christoph Bosshard, membre du Comité central de la FMH, responsable du département Données, démographie et qualité I Académie suisse pour la qualité en médecine (ASQM) 